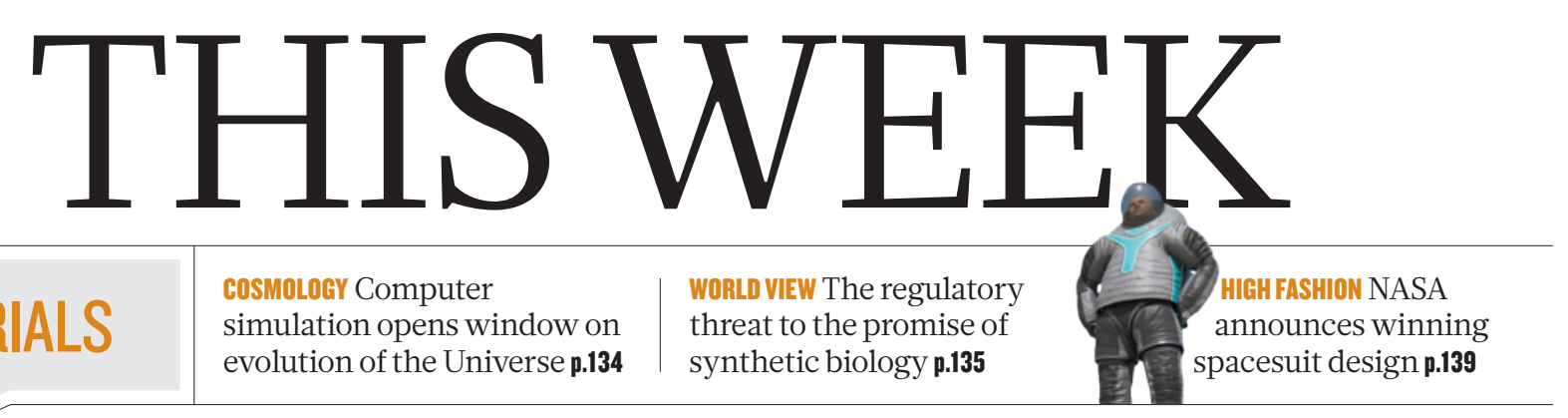

\section{EDITORIALS} evolution of the Universe $\mathbf{p . 1 3 4}$

threat to the promise of synthetic biology $\mathbf{p} .135$ pacesuit design $\mathbf{p} \mathbf{1 3 9}$

\title{
Tribal gathering
}

\author{
Before they can construct a cell, researchers in synthetic biology must first \\ build bridges between disciplines.
}

A lthough '-ology' denotes a subject of study, synthetic biology is more about building than observation. The field's founding papers were published just 14 years ago, but the scientists who call themselves synthetic biologists already have disparate goals. By many accounts, 'synbio' is less a coherent discipline than a collection of tribes under the same name. They do not interact with each other nearly enough.

Some synthetic biologists design genetic circuits to make microbes do fun or useful tricks - blink in sync, count pulses of chemicals, pump out biofuels or detect environmental pollutants. Others revise the chemistry of life's construction materials, crafting DNA and proteins that contain non-natural building blocks. Still others stitch together stretches of DNA to create whole chromosomes. In 2010, scientists replaced a bacterium's genome with a synthetic replica. In 2014 , a team fabricated a next-generation yeast chromosome, dropping cumbersome sequences and inserting convenient ones.

And those who influence the field with calls for funding and regulation are also driven by different goals. They agree that the outcomes of synthetic biology cannot yet be predicted, but some put caution foremost, seeking to avert any disasters that might result from an engineered microbe, whereas others focus on rewards, imagining revolutions in energy, medicine, nutrition or manufacturing.

So far, the field has avoided many of the public-image pitfalls that beleaguered genetically modified organisms (GMOs). Today, the term GMO is identified more with patented crops that help agriculture companies to sell products than with potential tools to grow food more efficiently or generate life-saving drugs. The best known application of synthetic biology, by contrast, is probably the manufacture of an accessible life-saving malaria medicine.

Although synthetic biologists need to address public concerns, they must also tend to the field from within, by fostering connections across disciplines. Divisions run deep. A synthetic biologist may come to a project as an iterative tinkerer, a methodical engineer or an intuitive explorer. Engineering types can be flummoxed by unpredictability, and sciencey types frustrated by demands for inflexible definitions and standards.

Some researchers have focused on establishing standards so that various 'gear parts' can be easily interchanged and combined. Others resist, arguing that variability among cells and circuits defies humandevised specifications. Some scientists push for open access, the better to mix and match inventions; others call for strong protections for intellectual property, the better to incentivize development of useful applications. The paucity of connection between these groups has provided ample fodder for sociologists of science.

As part of this week's special issue, a News Feature on page 152 suggests a way forward: open access and the safe-guarding of intellectual property could work together in a 'diverse ecology' in which circuit

parts are freely available and built-up components are eligible for patenting. Other gaps could be filled by technological and cultural innovations. Streamlined techniques can now characterize components across many contexts, including different cell types and combinations. These are helping to bring some predictability to the bewildering variability that is inherent in a living cell. Tellingly, one of the best known synthetic-biology companies, Amyris in Emeryville, California, combines randomized and rational approaches. It screens both randomly generated yeast mutants and designed strains every week to incrementally improve yields of a chemical product.

Synthetic biologists suggest that the various camps fail to connect because of apathy and habit rather than outright hostility. Whatever the reason, the discipline suffers from its divisions. Asked how to overcome the obstacles facing synthetic biology, experts call

"There will probably always be many tribes in synthetic biology, but there is reason to believe the field can avoid tribalism." ensconced in a specialty. Perhaps they will bring new and useful kinds
of thinking into the field.

Establishment of an international synthetic-biology society might be another way to foster productive mingling while allowing the field to maintain itself as a coherent discipline. Some encouraging developments have been evident at recent conferences, with panels comprising members from various camps. And more research is needed to learn how, for instance, intellectual-property and open-access schemes can be combined to boost innovation.

Although there will probably always be many tribes in synthetic biology, there is reason to believe that the field can avoid tribalism. Indeed, it is now more vital than ever that synthetic biologists present a united front. As Volker ter Meulen, co-chairman of IAP - the Global Network of Science Academies - writes in a World View on page 135, storm clouds are gathering on the horizon. Not everyone agrees that synthetic biology is a force for good, and that opposition has found its voice in a consultation for the global Convention for Biological Diversity. It is crucial, says ter Meulen, that the balancing voice of science is heard before false assumptions lead to the creation of onerous and unnecessary regulation. Everyone can agree on that. $\mathbf{~}$ 